\title{
Cholesterol-Dependent Kinase Activity Regulates Transmitter Release from Cerebellar Synapses
}

\author{
Alex J. Smith, ${ }^{1}$ Shuzo Sugita, ${ }^{1,2}$ and Milton P. Charlton ${ }^{1}$ \\ ${ }^{1}$ Department of Physiology, University of Toronto, Toronto, Ontario M5S 1A8, Canada, and ${ }^{2}$ Toronto Western Research Institute, University Health \\ Network, Toronto, Ontario M5T 2S8, Canada
}

\begin{abstract}
Changes in membrane cholesterol content can alter protein kinase activity, however, it is not known whether kinases regulating transmitter release are sensitive to membrane cholesterol content. Here we have used the cholesterol extracting agent methyl- $\beta$-cyclodextrin to measure the effects of acute cholesterol reduction on transmitter release from cultured cerebellar neurons. Cholesterol depletion increased the frequency of spontaneous transmitter release without altering the amplitude and time course of mEPSCs. Evoked transmitter release was decreased by cholesterol extraction and the paired pulse ratio was also decreased. Alterations in synaptic transmission were not associated with failure of action potential generation or changes in presynaptic $\mathrm{Ca}^{2+}$ signaling. Both the increase in $\mathrm{mEPSC}$ frequency and the change in paired pulse ratio were blocked by the broad spectrum protein kinase inhibitor staurosporine. The increase in mEPSC frequency was also sensitive to selective inhibitors of PKC and PKA. Our results therefore demonstrate that the activity of presynaptic protein kinases that regulate spontaneous and evoked neurotransmitter release is sensitive to changes of membrane cholesterol content.
\end{abstract}

\section{Introduction}

Reductions in the cholesterol content of neuronal membranes have been observed under conditions that include maturation of neurons in culture (Prinetti et al., 2001) and pathological disruption of cholesterol trafficking in Niemann-Pick C1 disease (Karten et al., 2002). Studies that have used the cholesterol binding oligosaccharide methyl- $\beta$-cyclodextrin $(\mathrm{M} \beta \mathrm{CD})$ to acutely reduce membrane cholesterol content have demonstrated that many of the molecules and processes involved in synaptic transmission are sensitive to cholesterol levels. For instance, cholesterol regulates the activity of voltage and $\mathrm{Ca}^{2+}$ gated $\mathrm{K}^{+}$channels that regulate action potential waveform and firing rate (Xia et al., 2004; Shmygol et al., 2007) and the function of $\mathrm{N}$ and P/Q type $\mathrm{Ca}^{2+}$ channels that supply the $\mathrm{Ca}^{2+}$ trigger for synaptic vesicle exocytosis at most synapses (Toselli et al., 2005; Davies et al., 2006). Cholesterol is required for the formation of synaptic vesicles (Thiele et al., 2000) and is required for t-SNARE clustering at vesicle docking sites in PC12 cells (Lang et al., 2001). Additionally, the localization or physical properties of neurotransmitter receptors including the NMDA receptor (Hering et al., 2003) are altered by changes in membrane cholesterol level.

Depletion of cholesterol from cortical synaptosomes reduces the amount of glutamate released by subsequent depolarization with elevated external $\mathrm{K}^{+}$(Taverna et al., 2004). In the intact

Received Dec. 22, 2009; revised March 22, 2010; accepted March 23, 2010.

This work was supported by Canadian Institutes of Health Research Operating Grants MOP-82827 to M.P.C. and MOP-57825 to S.S. We thank Dr. Rodney Tweten (University of Oklahoma, Oklahoma City, OK) and Dr. Bulent Matus (University of Windsor, Windsor, ON, Canada) for providing Perfringolysin 0 toxin D4 domain CDNA. We also thank Drs. L.Y. Wang, J.S. Dason, and R.G. Tsushima for critically reading the manuscript.

Correspondence should be addressed to Milton P. Charlton, Department of Physiology, MSB3308 1 King's College Circle, Toronto, Ontario M5S 1A8, Canada. E-mail: milton.charlton@utoronto.ca.

DOI:10.1523/JNEUROSCI.0170-10.2010

Copyright $\odot 2010$ the authors $\quad 0270-6474 / 10 / 306116-06 \$ 15.00 / 0$ synapses of crayfish neuromuscular junctions (Zamir and Charlton, 2006) and of cultured mouse hippocampal neurons (Wasser et al., 2007) cholesterol extraction reduces the amplitude of evoked synaptic responses but by different mechanisms. At the crayfish neuromuscular junction cholesterol depletion causes a failure in action potential transmission to release sites; when stimulation is applied focally at the release site the postsynaptic response is increased (Zamir and Charlton, 2006). In cultured hippocampal synapses cholesterol reduces the size of the readily releasable pool of vesicles (Wasser et al., 2007). Surprisingly, cholesterol depletion at the crayfish neuromuscular junction increases the frequency of spontaneous neurotransmitter release even after presynaptic injection of the $\mathrm{Ca}^{2+}$ chelator BAPTA. Therefore, cholesterol depletion increases spontaneous transmitter release through a $\mathrm{Ca}^{2+}$ independent mechanism (Zamir and Charlton, 2006).

Cholesterol depletion can lead to activation of several protein kinases that are known regulators of neurotransmitter release (Burgos et al., 2004; Cabrera-Poch et al., 2004). Functional cerebellar impairment is associated with impaired cholesterol trafficking in Niemann-Pick C1 disease so we have used a cerebellar culture model system to determine whether the activity of kinases regulating neurotransmitter release in the cerebellum is sensitive to changes in membrane cholesterol content.

\section{Materials and Methods}

Cell culture. All procedures were performed in accordance with animal welfare guidelines at the University of Toronto and were approved by the institutional animal care and use committee. Cerebellar cultures were prepared from embryonic day 17-18 mice according to a published protocol (Furuya et al.,1998) with modifications. Dissected cerebella were digested in $0.25 \%$ trypsin/EDTA (Invitrogen) for $15 \mathrm{~min}$ at $37^{\circ} \mathrm{C}$ then dissociated, filtered through a cell strainer, centrifuged at $240 \times g$ for 5 min, resuspended in plating solution (DMEM/F12, 10\% fetal bovine serum, $1 \times$ L-glutamine, $1 \times$ Penn/Strep) and plated at a density of 2500 
A

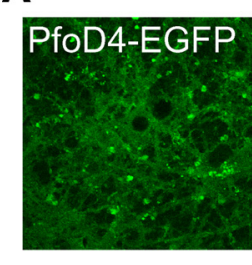

B
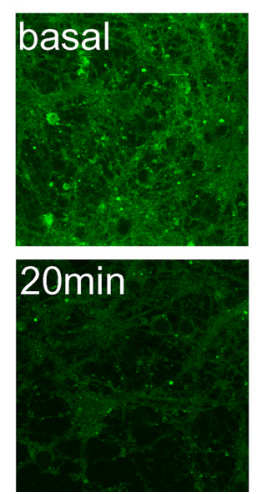

C
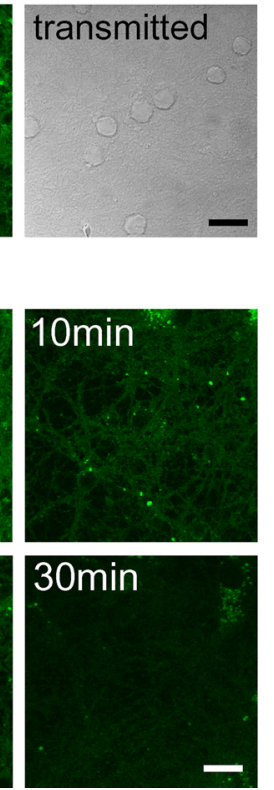

D
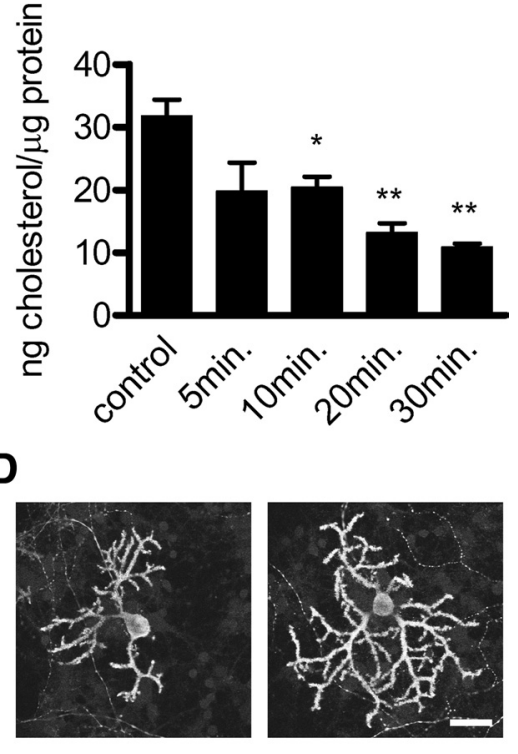

Figure 1. Treatment with $M \beta C D$ causes extensive reduction of membrane cholesterol content. $\boldsymbol{A}$, Distribution of lipid rafts in cultured cerebellar neurons labeled with Pf0-D4-EGFP (left); transmitted light image (right). $\boldsymbol{B}$, Cholesterol depletion following exposure to $5 \mathrm{~mm} \mathrm{M} \beta C D$ for the indicated durations is demonstrated by reduced PfO-D4-EGFP binding. Scale bars: $A, B, 10 \mu \mathrm{m}$. $\boldsymbol{C}$, Cholesterol content of cell lysates quantified by Amplex Red cholesterol assay under control conditions and following $M \beta C D$ treatment for the indicated periods; data were normalized to the lysate protein concentration. Bars show the mean and SEM of experiments performed in triplicate; ${ }^{*} p<0.05,{ }^{* *} p<0.01$ versus control by $t$ test. $D$, Calbindin D28K staining of cultured Purkinje cells under control conditions (left) and following exposure to $5 \mathrm{~mm} \mathrm{M \beta CD}$ for $20 \mathrm{~min}$ (right). Scale bar, $20 \mu \mathrm{m}$.

cells/ $\mathrm{mm}^{2}$ on $12 \mathrm{~mm}$ coverslips coated with poly-D-lysine $(70-150 \mathrm{kDa}$; Sigma). Cells were incubated at $37^{\circ} \mathrm{C}$ for $3 \mathrm{~h}$ then $450 \mu \mathrm{l}$ of culture media (DMEM/F12, 2\% B27 supplements, 2 mM L-glutamine, $1 \times$ Penn/Strep; all Invitrogen) was added per well. Experiments were performed on cells after $10-16 \mathrm{~d}$ in vitro.

Confocal fluorescence microscopy and cholesterol quantitation. Cells on coverslips were treated as indicated in the figure legend, rinsed in PBS then fixed in $4 \%$ formaldehyde, permeabilized and stained sequentially with anti-calbindin D28K (Millipore) and Alexa 594 anti-rabbit (Invitrogen) antibodies. For cholesterol staining, the D4 domain of Perfringolysin $O$ (PfO) toxin (Ohno-Iwashita et al., 1990) and emerald-GFP were amplified and ligated behind GST into modified pGEX-KG vector then transfected into competent BL21(DE3) E. coli; bacterial growth, induction and protein purification were performed using standard procedures. The recombinant protein was applied at $250 \mu \mathrm{g} / \mathrm{ml}$ for $10 \mathrm{~min}$ following fixation without a permeabilization step. Coverslips were mounted on microscope slides in ProLong Gold reagent (Invitrogen), images were captured with a Leica TCS SL confocal microscope. Biochemical measurement of cholesterol was performed using the Amplex Red Cholesterol Assay Kit (Invitrogen) according to the manufacturer's instructions, without cholesterol esterase treatment. Resorufin product was imaged in 96 well plates with a CCD-based fluorescent scanner (Ettan DIGE Imager; GE Healthcare) and quantified with ImageJ software. Protein concentration of lysates was measured spectrophotometrically using Bradford Reagent (Sigma) with BSA standards.

Electrophysiology. Experiments were performed in HEPES-buffered saline (HBS) containing the following (in mM): $140 \mathrm{NaCl}, 10$ glucose, 3-5 $\mathrm{KCl}, 2 \mathrm{CaCl}_{2}, 1 \mathrm{MgCl}_{2}, 5$ sucrose, $10 \mathrm{HEPES}, \mathrm{pH} 7.35$, at $25^{\circ} \mathrm{C}$, and cells were allowed to equilibrate to this buffer for at least $30 \mathrm{~min}$ before the start of experiments. Coverslips with adherent cultured neurons were placed in a perfusion chamber on an upright microscope and viewed under a $20 \times, 0.4$ numerical aperture (NA) water-immersion objective. Solutions were exchanged by gravitational flow at a rate of $0.5-1.0 \mathrm{ml} /$ min. For recording of synaptic currents, Purkinje cells were visually identified on the criteria of large soma, highly branched processes and prominent nucleus and were placed under whole-cell voltage clamp at holding potential of $-75 \mathrm{mV}$ using 2.5-3.5 $\mathrm{M} \Omega$ electrodes containing the following (in $\mathrm{mm}$ ): $140 \mathrm{CsCl}, 10$ HEPES, 5 EGTA, 2 MgATP [pH adjusted to 7.35 with $\mathrm{CsOH}$; corrected for liquid junction potential (LJP) of $6 \mathrm{mV}$ ]. Picrotoxin, $50 \mu \mathrm{M}$, was present for all experiments and $0.5 \mu \mathrm{M}$ tetrodotoxin (Alomone Labs) was present for mEPSC recordings. Recording instrumentation consisted of an AM Systems model 2400 patch-clamp amplifier and a National Instruments PCI 6024E digital I/O board under the control of an Electronic Data Recorder or Whole Cell Program (Strathclyde Electrophysiology Software). Output current signals were low pass filtered at $5 \mathrm{kHz}$ and digitized at 10 $\mathrm{kHz}$. Initial series resistance values were between 8 and $20 \mathrm{M} \Omega$ and were checked at $5 \mathrm{~min}$ intervals during recording; series resistance was not compensated. Recordings were excluded from analysis if holding current exceeded $250 \mathrm{pA}$ or series resistance varied by $>30 \%$ during the experiment. A saline filled patch electrode or $\theta$-glass electrode with the tip placed immediately above the soma of a granule neuron was used for extracellular stimulation. Cell pairs were selected for responses displaying facilitation to paired pulses at $20 \mathrm{~Hz}$. Currentclamp recordings from granule neurons were performed with electrodes of 5-7 M $\Omega$ and intracellular buffer containing the following (in $\mathrm{mM}$ ): $140 \mathrm{~K}$-gluconate, $10 \mathrm{KCl}, 10 \mathrm{HEPES}, 5$ EGTA, 2 MgATP ( $\mathrm{pH}$ adjusted to 7.35 with $\mathrm{KOH}$; recordings compensated for LJP of $15 \mathrm{mV}$ ).

$\mathrm{Ca}^{2+}$ imaging. Coverslips were incubated in control saline or in saline containing $5 \mathrm{~mm} \mathrm{M} \beta \mathrm{CD}$ for $30 \mathrm{~min}$. Granule neurons were then voltageclamped at $-70 \mathrm{mV}$ using whole-cell electrodes containing (in mM) 140 K-gluconate, $10 \mathrm{KCl}, 10 \mathrm{HEPES}, 2 \mathrm{MgATP}$, and 0.2 fura-2, and dialyzed for $10 \mathrm{~min}$ to allow indicator diffusion along the proximal axon segment. Imaging equipment consisted of a Polychrome V monochromator (TILL Photonics), Ixon DU897 EM-CCD camera (Andor Technology) operated in conventional readout mode with $2 \times 2$ pixel binning $(0.8 \mu \mathrm{m} \times$ $0.8 \mu \mathrm{m})$ and an upright Nikon microscope with $40 \times, 0.7$ NA Olympus water dipping lens. Image acquisition was controlled with WinFluor software (Strathclyde Electrophysiology Software). Ratio pairs (360/380 nm excitation) were acquired at $2 \mathrm{~Hz}$ while an action potential-like stimulus train consisting of $1 \mathrm{~ms}$ voltage steps to $+30 \mathrm{mV}$, repeated 20 times at 20 $\mathrm{Hz}$, was applied. Ratio images were generated using a cutoff of 20 intensity levels above background at both wavelengths. Presynaptic ROIs were readily identifiable from cellular morphology (see Fig. $3 D$ ) and consisted of $2-8$ contiguous pixels. Mean ratio values from these ROIs were converted to $\left[\mathrm{Ca}^{2+}\right]_{\mathrm{i}}$ values with $R_{\min }$ and $R_{\max }$ values calculated from in vitro calibration solutions containing 0 or $39.8 \mu \mathrm{M}$ free $\mathrm{Ca}^{2+}$ (Invitrogen), a viscosity correction factor of 0.8 and an assumed $K_{\mathrm{d}}$ of $224 \mathrm{~nm}$.

Data analysis. Measurement of EPSC peak amplitude and timing was performed with Clampfit software (Molecular Devices); AP and afterhyperpolarization (AHP) amplitude were defined as the maximum and minimum amplitude changes occurring within $10 \mathrm{~ms}$ of the visually identified onset of the AP. mEPSC amplitude and frequency were measured with Mini Analysis software (Synaptosoft). Graphing and statistical analysis of results was performed with GraphPad Prism software.

\section{Results}

Acute depletion of cholesterol from cultured cerebellar neurons The synapses formed between granule and Purkinje neurons in vitro allow a defined, physiologically relevant synaptic connection to be studied in a dissociated cell monolayer (Hirano et al., 
1986). To visualize the effect of $\mathrm{M} \beta \mathrm{CD}$ treatment on cholesterol distribution in this preparation we used the cholesterol binding D4 domain of Perfringolysin O. (PfO) toxin, a marker of lipid raft domains (Waheed et al., 2001). Under basal conditions D4 domain staining revealed a pattern of bright spots and more diffuse membrane staining (Fig. $1 A$ ). Treatment with $5 \mathrm{~mm} \mathrm{M} \beta C D$ caused a progressive reduction in the intensity of $\mathrm{D} 4$ domain staining (Fig. $1 \mathrm{~B}$ ), demonstrating that lipid rafts were dispersed by $M \beta C D$. To quantitate the amount of cholesterol extracted we used the Amplex Red cholesterol assay; this demonstrated that $\mathrm{M} \beta \mathrm{CD}$ treatment caused a progressive decline in cholesterol content to $<50 \%$ of the initial value after $30 \mathrm{~min}$ (Fig. $1 C$ ). We also investigated the possibility that cholesterol depletion compromised membrane integrity. Results in Figure $1 D$ demonstrate that this was not the case as the morphology of Purkinje cells visualized by Calbindin D28K staining was similar between control treatment and 20 min treatment with $5 \mathrm{~mm} \mathrm{M} \beta C D$.

\section{Effect of cholesterol depletion on synaptic transmission}

We recorded synaptic responses from Purkinje cells as cholesterol was extracted with $5 \mathrm{~mm} M \beta C D$. This treatment caused a progressive increase in the frequency of mEPSCs over the course of $\mathrm{M} \beta \mathrm{CD}$ exposure (Fig. $2 A, B$ ). Treatment with $5 \mathrm{~mm} \mathrm{M} \beta \mathrm{CD}$ precomplexed with $0.25 \mathrm{~mm}$ cholesterol did not increase mEPSC frequency, demonstrating a requirement for cholesterol extraction (Fig. $2 B$ ). The mean amplitude, time course and cumulative amplitude distribution of mEPSCs were unchanged by $\mathrm{M} \beta \mathrm{CD}$ treatment (Fig. 2C,D) demonstrating that quantal size was unaffected under the conditions we used. It is therefore unlikely that there were major postsynaptic effects in our experiments but there was a significant increase in spontaneous vesicular release probability.

To determine the effect of reducing membrane cholesterol content on evoked synaptic transmission we recorded EPSCs from Purkinje cells generated by extracellular stimulation of granule neurons (Fig. 2 E). Stimulus pulses were delivered at 0.05 $\mathrm{Hz}$, EPSCs had mean basal amplitude of $203 \pm 38 \mathrm{pA}$ and the mean amplitude of EPSCs was stable in normal saline. Treatment with $5 \mathrm{mM} \mathrm{M} \beta C D$ reduced EPSC amplitude (Fig. $2 E, F$ ) and as the mEPSC amplitude was unchanged, this result demonstrates that the number of vesicles released per stimulus was reduced by cholesterol depletion. However, when $5 \mathrm{~mm} \mathrm{M} \beta \mathrm{CD} / 0.25 \mathrm{~mm}$ cholesterol complex was applied there was no change in EPSP amplitude. This indicated that $\mathrm{M} \beta \mathrm{CD}$ had minimal nonspecific effects on EPSP amplitude. EPSC kinetics were not significantly different in these experiments, the delay from stimulus onset to EPSC peak was $3.46 \pm 0.12 \mathrm{~ms}$ under basal conditions and $3.83 \pm$ $0.25 \mathrm{~ms}$ following treatment with $\mathrm{M} \beta \mathrm{CD}$ ( $p=0.098$ by paired $t$ test). The decay time constant of single exponentials fitted to the falling portion of the EPSC was $1.41 \pm 0.19 \mathrm{~ms}$ under basal conditions and $1.52 \pm 0.23 \mathrm{~ms}$ following $\mathrm{M} \beta \mathrm{CD}$ treatment $(p=0.60$ by paired $t$ test).

\section{Presynaptic action potentials and $\mathrm{Ca}^{2+}$ signaling}

As discussed in the introduction, the activity of many $\mathrm{K}^{+}$and $\mathrm{Ca}^{2+}$ channels is sensitive to membrane cholesterol levels so we investigated the possibility that changes in action potential transmission or in presynaptic $\mathrm{Ca}^{2+}$ influx caused the changes in synaptic currents we observed following cholesterol depletion. We used the whole-cell current-clamp configuration to record action potentials in cultured granule neurons. Holding current was applied to maintain cells at $-85 \mathrm{mV}$ and brief $(50 \mathrm{~ms})$ pulses of depolarizing current injection of sufficient amplitude (15-30 pA)
A

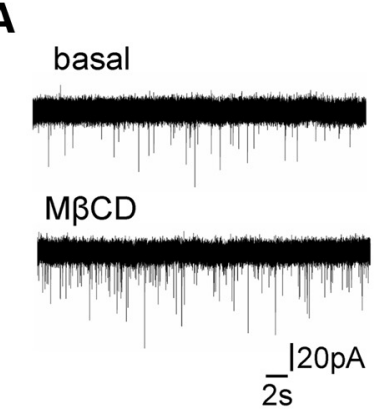

B

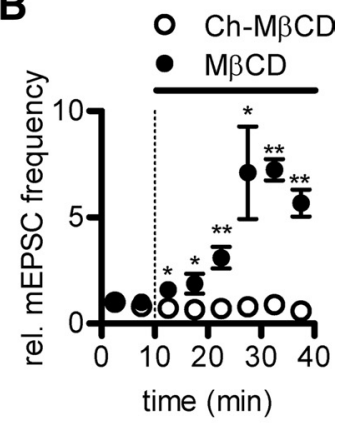

C

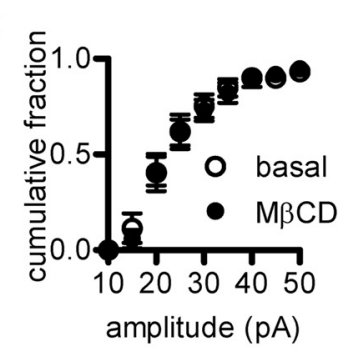

D

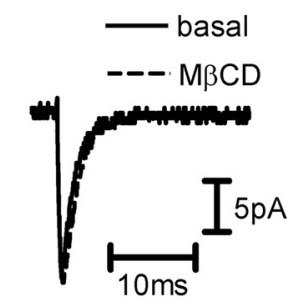

E
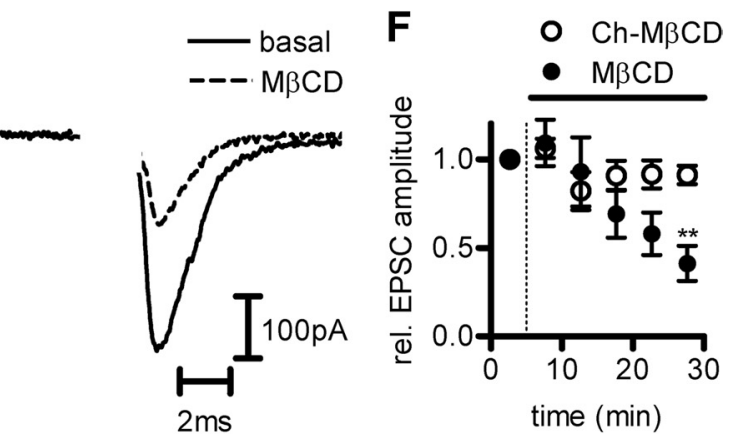

Figure 2. Cholesterol depletion alters synaptic transmission. $\boldsymbol{A}$, Representative whole-cell recording of $\mathrm{mEPSC}$ from a Purkinje neuron under basal conditions and after $25 \mathrm{~min}$ exposure to $5 \mathrm{~mm} \mathrm{M} \beta C D$. $\boldsymbol{B}$, Relative mEPSC frequency during exposure to $5 \mathrm{~mm} \mathrm{M} \beta C D$ ( $n=5$ cells, closed circles) or $5 \mathrm{~mm}$ cholesterol-M $\beta C D$ complex ( $n=5$ cells, open circles). mEPSC frequency was calculated for $5 \mathrm{~min}$ bins for $5 \mathrm{~min}$ before and $30 \mathrm{~min}$ after exposure and then normalized to the basal frequency during the first 5 min of recording from each cell ( $n=5$ cells). C, Cumulative amplitude distribution of $\mathrm{mEPSC}$ in $5 \mathrm{pA}$ bins. The fraction of events falling within each amplitude bin was determined for the cells from the experiment shown in B during a 5 min basal period (open circles) or a 5 min period between 25 and 30 min of exposure to $5 \mathrm{~mm} \mathrm{M \beta CD}$ (closed circles). Fractions were plotted as mean and SE of the 5 cells. D, Average of all mEPSCs recorded from a single experiment during a $5 \mathrm{~min}$ basal period (solid line) and a $5 \mathrm{~min}$ period following 25 min exposure to $5 \mathrm{~mm} \mathrm{M \beta CD}$ (dotted line). $\boldsymbol{E}$, EPSCs recorded from a granule neuron-Purkinje neuron pair under basal conditions and following 25 min of perfusion with 5 тм $M \beta C D$. Stimulus artifacts were removed for clarity. $\boldsymbol{F}$, Time course of the change in peak amplitude of the EPSC during exposure to $5 \mathrm{~mm} \mathrm{M} \beta C D$ (closed circles, $n=6$ ) or $5 \mathrm{~mm}$ cholesterol-M $\beta C D$ (open circles, $n=5$ ) from 5 min of recording onwards. Data points represent the mean and SE of the normalized EPSP amplitude. ${ }^{*} p<0.05,{ }^{* *} p<0.01$ versus control by $t$ test.

to induce single action potentials were applied at $20 \mathrm{~s}$ intervals (Fig. 3A). M $\beta C D$ treatment caused an increase in AP amplitude and loss of afterhyperpolarization (Fig. $3 B, C$ ). Resting membrane potential was not affected by $\mathrm{M} \beta \mathrm{CD}$ treatment $(-86.2 \pm$ $1.6 \mathrm{mV}$ basal; $-86.5 \pm 1.0 \mathrm{mV} 20 \mathrm{~min} \mathrm{M} \beta \mathrm{CD} ; n=4$ each). Somatic action potential generation was therefore not blocked by cholesterol depletion. To test the possibility that action potential propagation or presynaptic $\mathrm{Ca}^{2+}$ influx were altered we measured residual $\mathrm{Ca}^{2+}$ accumulation in presynaptic terminals generated in response to somatic action potential-like voltage pulses by ratiometric imaging of fura-2 (Fig. $3 D$ ). Presynaptic terminals were visually identified as swellings along the thin axon and dis- 
A

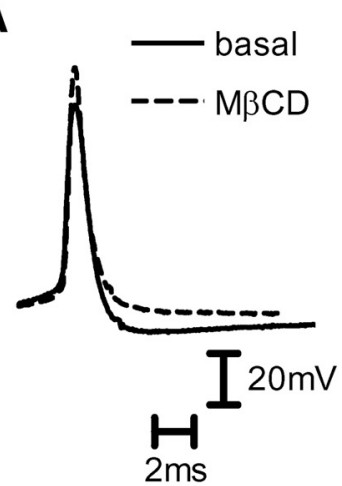

B

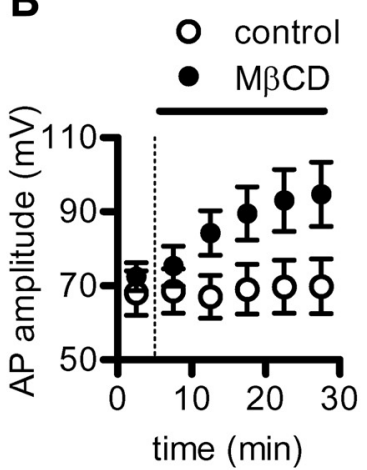

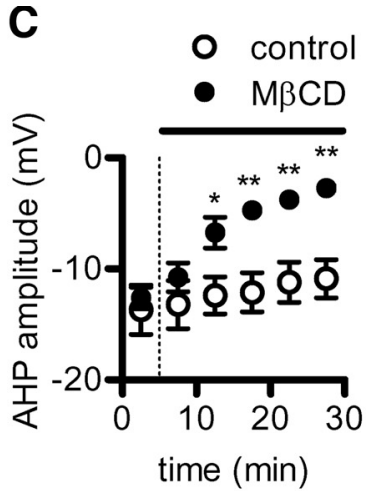

D

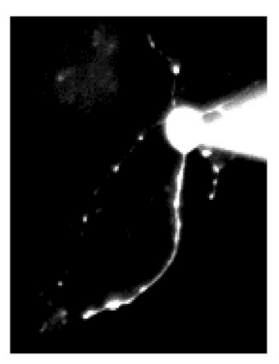

E

$\mathbf{F}$

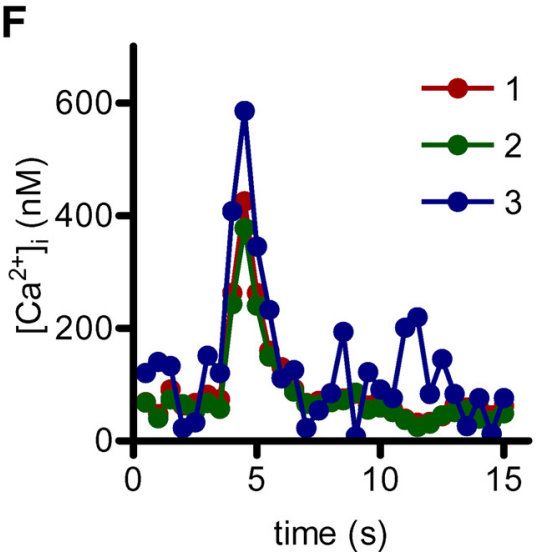

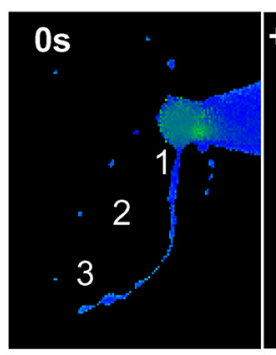
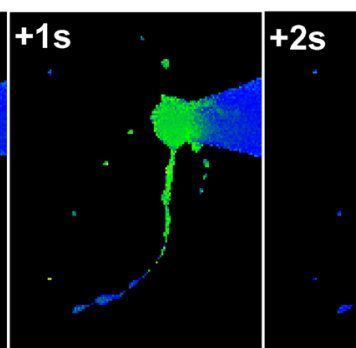

G

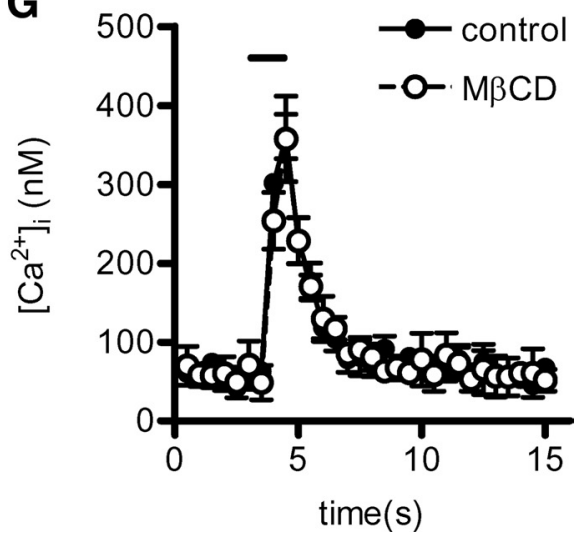

Figure 3. Alterations to presynaptic excitability and $\mathrm{Ca}^{2+}$ signaling do not explain the effects of cholesterol depletion on synaptic transmission. $A$, Representative action potential recordings from a granule neuron subjected to depolarizing current injection ( $+20 \mathrm{pA}, 50 \mathrm{~ms}$ ) under basal conditions and following 25 min exposure to $5 \mathrm{~mm} \mathrm{M} \beta C D$. $\boldsymbol{B}$, Alteration in AP amplitude for neurons held in $5 \mathrm{~mm} \mathrm{M} \beta C D$ for 25 min (black bars) or held in control saline for 25 min (open bars). C, Same as $\boldsymbol{B}$ but with measurement of AHP amplitude. $\boldsymbol{D}$, Granule neuron under whole-cell voltage clamp with intracellular solution containing $200 \mu \mathrm{m}$ fura- 2 imaged with $380 \mathrm{~nm}$ excitation light. Scale bar, $10 \mu \mathrm{m}$. $\boldsymbol{E}$, Ratio images of $\left[\mathrm{Ca}^{2+}\right]_{\mathrm{i}}$ in a granule cell stimulated with 20 pulses at $20 \mathrm{~Hz} . \boldsymbol{F},\left[\mathrm{Ca}^{2+}\right]_{i}$ was measured from areas corresponding to the presynaptic boutons labeled $\mathbf{1 - 3}$ in $\boldsymbol{E}$. G, Mean and SE of responses measured under control conditions ( $n=5$ cells) or from neurons pretreated with $5 \mathrm{~mm} M \beta C D$ for $30 \mathrm{~min}(n=3$ cells).

played large $\mathrm{Ca}^{2+}$ increases in response to a train of 20 voltage pulses (Fig. 3D-F). No difference in the amplitude or kinetics of these responses was detected when measurements were made from cells that had been treated for 30 min with $5 \mathrm{~mm} \mathrm{M} \beta C D$ (Fig. $3 G$ ). These results demonstrate that the decrease in EPSC amplitude observed following $\mathrm{M} \beta \mathrm{CD}$ treatment is not a consequence of reduction in presynaptic $\mathrm{Ca}^{2+}$ influx. As basal $\left[\mathrm{Ca}^{2+}\right]$ was not different in the terminals of cholesterol depleted cells, the increase in mEPSC frequency observed following cholesterol depletion occurred by a $\mathrm{Ca}^{2+}$ independent mechanism.

\section{Cholesterol depletion and presynaptic kinase activity}

In many synapses spontaneous transmitter release is controlled in part by the activity of various protein kinases and the activity of some of these kinases might be sensitive to changes of membrane cholesterol content. We used a broad-spectrum protein kinase inhibitor, staurosporine, to determine whether kinases regulating neurotransmitter release were activated by cholesterol depletion. Pretreatment with staurosporine for $10 \mathrm{~min}$ prevented the increase of mEPSC frequency caused by subsequent $\mathrm{M} \beta \mathrm{CD}$ treatment (Fig. $4 A, C)$. Treatment with staurosporine by itself did not cause a significant decrease in mEPSC frequency (Fig. $4 B$ ) suggesting that basal phosphorylation turnover rate is low and the effect of $M \beta C D$ is to increase the activity of a staurosporinesensitive kinase rather than to block a phosphatase. To determine the identity of kinases mediating the increase in mEPSC frequency we assessed the ability of selective PKC and PKA inhibitors to block the mEPSC frequency increase observed following cholesterol depletion. Pretreatment with the PKA selective inhibitor Rp-cAMPS $(100 \mu \mathrm{M})$ or with the PKC selective inhibitor GF109203X (1 $\mu \mathrm{M})$ significantly reduced the extent of the increase in mEPSC frequency observed following cholesterol extraction when compared with control experiments performed in parallel (Fig. 4D,E). Kinase activation can also influence evoked release and can change the paired pulse ratio at parallel fiber Purkinje cell synapses in cerebellar slices (Salin et al., 1996). We investigated the effect of staurosporine pretreatment on paired pulse ratio using paired stimulation at $20 \mathrm{~Hz}$. The first response was still inhibited by $\mathrm{M} \beta \mathrm{CD}$ in cultures pretreated with staurosporine (Fig. $4 F, G$ ). The reduction in paired pulse ratio was not observed when staurosporine pretreated cultures were subject to cholesterol depletion with $\mathrm{M} \beta \mathrm{CD}$ (Fig. $4 H$ ), demonstrating that this change was also a consequence of increased kinase activity following cholesterol depletion. Our results therefore demonstrate that kinases regulating both spontaneous and evoked neurotransmitter release are sensitive to changes in membrane cholesterol content and that the effects of cholesterol depletion on synaptic transmission can be partly explained by increased presynaptic kinase activity.

\section{Discussion}

We have shown that cholesterol content controls synaptic transmission between cultured cerebellar neurons. The rate of spontaneous neurotransmitter release is increased following cholesterol depletion by a mechanism requiring activation of presynaptic protein kinases. The amplitude of evoked synaptic responses is decreased consistent with a decrease in the size of the readily releasable pool of vesicles following cholesterol depletion. 

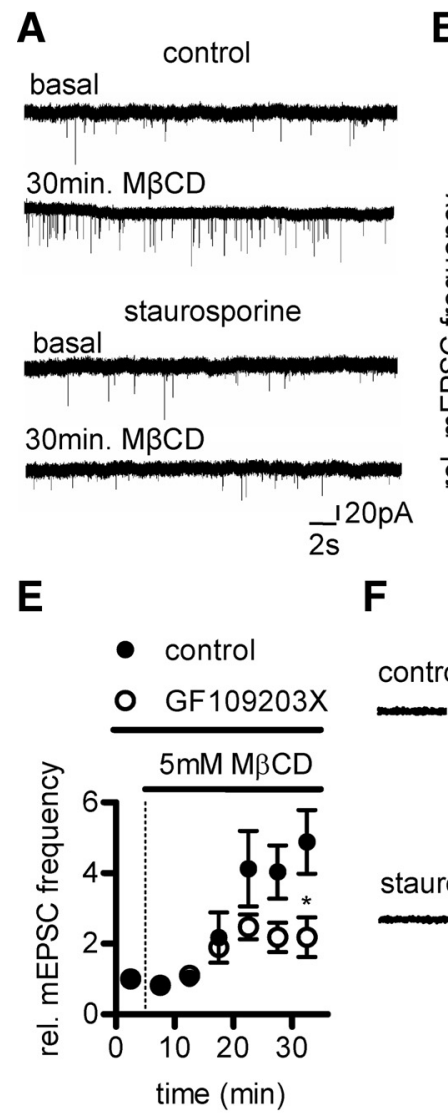

B

$\mathbf{F}$

$\mathbf{F}$

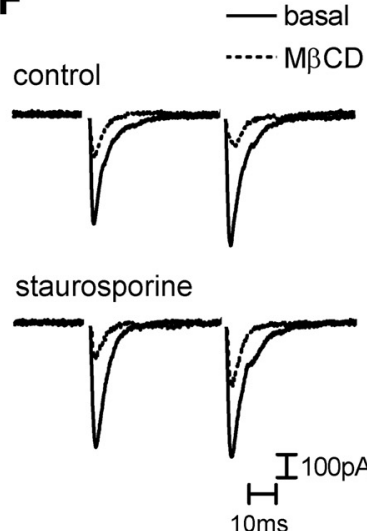

C
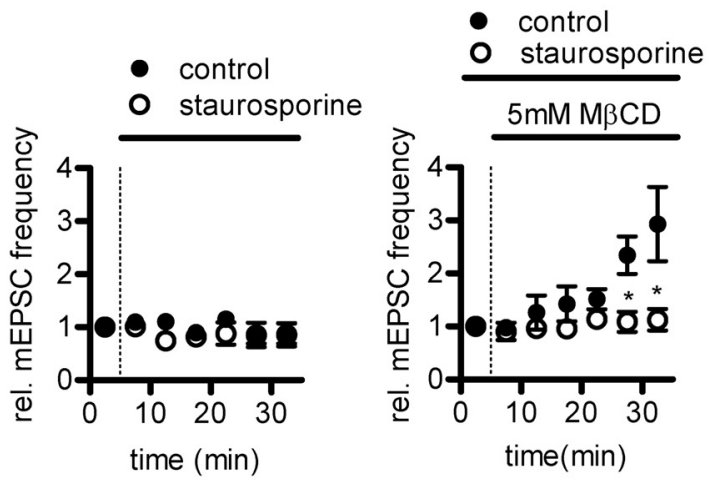

G

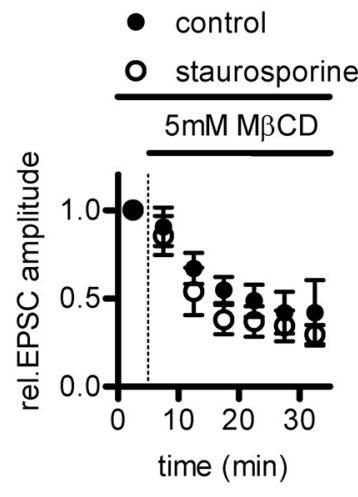

D

- control

- Rp-cAMPS

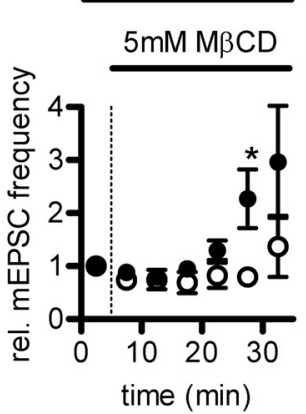

H

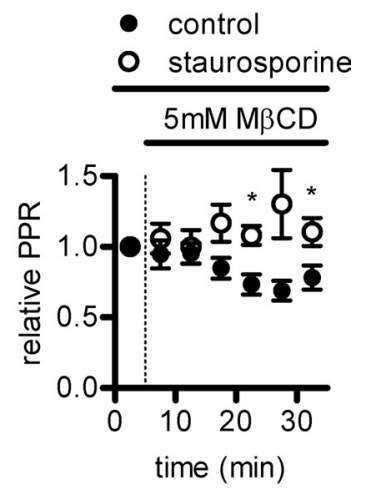

Figure 4. Protein kinase activation mediates some of the effects of cholesterol depletion. $\boldsymbol{A}, \mathrm{mEPSC}$ recordings from cells before and after $\mathrm{M} \beta C \mathrm{C}$ treatment under control conditions (top) or with $1 \mu \mathrm{m}$ staurosporine pretreatment (bottom). $\boldsymbol{B}$, Effect of $1 \mu \mathrm{m}$ staurosporine addition on relative mEPSC frequency (open circles, $n=4$ ) and control recordings with no drug addition (closed circles, $n=4)$. C, Mean and SE of the relative mEPSC frequency during $M \beta C D$ wash in under control conditions (filled circles, $n=6$ ) or following pretreatment with $1 \mu \mathrm{m}$ staurosporine for at least 10 min before the start of recording (open circles, $n=5$ ). $\boldsymbol{D}$, Relative mEPSC frequency during M $\beta C D$ treatment under control conditions or with $100 \mu \mathrm{m}$ Rp-CAMPS pretreatment ( $n=4$ each). $\boldsymbol{E}$, Relative mEPSC frequency during M $\beta C D$ treatment under control conditions (filled circles $n=6$ ) or with $1 \mu \mathrm{m}$ GF109203X pretreatment (open circles, $n=6$ ). $F$, Paired EPSCs recorded from Purkinje cells before and after $M \beta C D$ treatment under control conditions (top panel) and with $1 \mu \mathrm{m}$ staurosporine pretreatment (bottom panel). G, Effect of M $\beta C D$ treatment on EPSC amplitude with or without treatment with $1 \mu \mathrm{m}$ staurosporine $10 \mathrm{~min}$ before application of $\mathrm{M} \beta C \mathrm{C}(n=5$ each). $\boldsymbol{H}$, Effect of $\mathrm{M} \beta C D$ treatment on paired pulse ratio with or without staurosporine pretreatment $(n=5$ each; ${ }^{*} p<0.05$ vs control by $t$ test in all panels).

Short term synaptic plasticity is also altered by cholesterol depletion, again by a kinase-dependent mechanism.

Cholesterol depletion is known to increase the activity of several staurosporine-sensitive kinases including PKA, PKC and src (Burgos et al., 2004; Cabrera-Poch et al., 2004). The mechanisms linking cholesterol levels to kinase activity are not fully understood. In MDCK cells $\mathrm{M} \beta \mathrm{CD}$ treatment activates PKA without increasing cAMP levels, possibly by disrupting inhibitory complexes localized to lipid rafts (Burgos et al., 2004). Activation of $\mathrm{PKC} \varepsilon$ and src in $\mathrm{M} \beta \mathrm{CD}$-treated PC12 cells is also thought to be a consequence of alterations to localized lipid domain interactions (Cabrera-Poch et al., 2004). In addition to direct effects on kinase activity, the activity of PP2A phosphatase is sensitive to cholesterol depletion (Wang et al., 2005), therefore phosphatase inactivation may also contribute to an increase in protein phosphorylation following $\mathrm{M} \beta \mathrm{CD}$ treatment. Activation of both PKC and PKA can increase mEPSC frequency (Carroll et al., 1998), similar kinases may mediate the effect of $M \beta C D$ on PPR as PKA activation decreases PPR at parallel fiber synapses in cerebellar slices (Salin et al., 1996). The precise nature of the phosphorylation event(s) linking cholesterol depletion to increased mEPSC frequency and decreased PPR are beyond the scope of this article but the most plausible mechanistic explanation is that lipid raft disruption allows active kinases access to some compo- nent of the release apparatus and subsequent phosphorylation increases release probability.

The reduction in EPSC amplitude observed following $\mathrm{M} \beta \mathrm{CD}$ treatment could be considered paradoxical given the effects on mEPSC frequency and PPR; however, cholesterol depletion has effects on vesicle recycling and release site structure that could explain this observation. Cholesterol depletion inhibits several forms of endocytosis (Parton and Richards, 2003) and biogenesis of synaptic like microvesicles in $\mathrm{PC} 12$ cells is more readily blocked by cholesterol depletion than bulk endocytosis (Thiele et al., 2000). Cholesterol is also required for clustering of the t-SNARE syntaxin in lipid raft membrane domains in PC12 cells; disruption of these domains with $\mathrm{M} \beta \mathrm{CD}$ inhibits dense core vesicle docking and subsequent exocytosis (Lang et al., 2001; Zhang et al., 2009). Consistent with these observations, cholesterol depletion decreases the number of synaptic vesicles and the size of the readily releasable pool of vesicles in cultured hippocampal neurons (Wasser et al., 2007). Cholesterol depletion also alters the kinetics of release of dense core vesicles from PC12 cells and may be important for fusion pore expansion during exocytosis of large vesicles (Zhang et al., 2009). We found that mEPSC amplitude and time course were unchanged following cholesterol extraction suggesting that the rate of release of glutamate from small synaptic vesicles is not sensitive to changes in membrane 
cholesterol content. Our results also suggest that the alterations in synaptic responses we observed were mainly caused by presynaptic effects; however, we cannot rule out the possibility that cholesterol depletion may have caused a subset of synapses to become postsynaptically silenced in addition to any presynaptic effects.

We investigated the possibility that alterations in action potential transmission or presynaptic $\mathrm{Ca}^{2+}$ influx contributed to the reduction of EPSC amplitude but found no significant differences in the amplitude or time course of $\mathrm{Ca}^{2+}$ transients in presynaptic boutons from $\mathrm{M} \beta \mathrm{CD}$-treated cells. The increased amplitude of somatic action potentials and reduction in the afterhyperpolarization amplitude are consistent with studies demonstrating regulation of voltage- and $\mathrm{Ca}^{2+}$-gated $\mathrm{K}^{+}$channels by the membrane lipid environment in general and by cholesterol specifically (Xia et al., 2004; Shmygol et al., 2007). These results demonstrate that changes in membrane cholesterol levels have effects on somatic excitability but that action potential transmission and presynaptic $\mathrm{Ca}^{2+}$ signaling are relatively resistant to cholesterol depletion.

The lipid composition of neuronal membranes is altered during cellular maturation and in some pathological conditions. In cerebellar neurons cultured from $8 \mathrm{~d}$ postnatal rats the molar ratio of cholesterol to glycerophospholipids decreases from 0.146 to 0.108 between 8 and 17 DIV (Prinetti et al., 2001). Conversely the cholesterol content of hippocampal neuronal membranes increases with time in culture (Nicholson and Ferreira, 2009). Our results suggest that changes in membrane cholesterol content could lead to alterations in presynaptic kinase signaling as neurons develop in vitro. Changes in cholesterol trafficking or metabolism have been implicated in the pathogenesis of several neurodegenerative conditions, most notably Niemann-Pick C1 disease which is caused by mutations of a gene involved in intracellular cholesterol trafficking; this causes cerebellar neurodegeneration (Sarna et al., 2003). A reduction in axonal cholesterol is observed in sympathetic neurons derived from mice deficient for the Niemann-Pick C1 (NPC1) protein (Karten et al., 2002). Interestingly, NPC1 pathology is associated with cytoskeletal damage and hyperphosphorylation of the microtubule binding protein tau (Bu et al., 2002); kinase activation caused by lipid raft disruption has been suggested as a cause of tau hyperphosphorylation (Sawamura et al., 2003). Our results demonstrate that similar mechanisms linking perturbations of membrane cholesterol content to kinase activation could contribute to synaptic dysfunction in neurodegenerative disease.

\section{References}

Bu B, Li J, Davies P, Vincent I (2002) Deregulation of cdk5, hyperphosphorylation, and cytoskeletal pathology in the Niemann-Pick type $\mathrm{C}$ murine model. J Neurosci 22:6515-6525.

Burgos PV, Klattenhoff C, de la Fuente E, Rigotti A, González A (2004) Cholesterol depletion induces PKA-mediated basolateral-to-apical transcytosis of the scavenger receptor class B type I in MDCK cells. Proc Natl Acad Sci U S A 101:3845-3850.

Cabrera-Poch N, Sánchez-Ruiloba L, Rodriguez-Martínez M, Iglesias T (2004) Lipid raft disruption triggers protein kinase $\mathrm{C}$ and src-dependent protein kinase D activation and Kidins 220 phosphorylation in neuronal cells. J Biol Chem 279:28592-28602.

Carroll RC, Nicoll RA, Malenka RC (1998) Effects of PKA and PKC on miniature excitatory postsynaptic currents in CA1 pyramidal cells. J Neurophysiol 80:2797-2800.

Davies A, Douglas L, Hendrich J, Wratten J, Tran Van Minh A, Foucault I, Koch D, Pratt WS, Saibil HR, Dolphin AC (2006) The calcium channel $\alpha_{2} \delta$-2 subunit partitions with CaV2.1 into lipid rafts in cerebellum: implications for localization and function. J Neurosci 26:8748-8757.

Furuya S, Makino A, Hirabayashi Y (1998) An improved method for culturing cerebellar Purkinje cells with differentiated dendrites under a mixed monolayer setting. Brain Res Brain Res Protoc 3:192-198.

Hering H, Lin CC, Sheng M (2003) Lipid rafts in the maintenance of synapses, dendritic spines, and surface AMPA receptor stability. J Neurosci 23:3262-3271.

Hirano T, Kubo Y, Wu MM (1986) Cerebellar granule cells in culture: monosynaptic connections with Purkinje cells and ionic currents. Proc Natl Acad Sci U S A 83:4957-4961.

Karten B, Vance DE, Campenot RB, Vance JE (2002) Cholesterol accumulates in cell bodies, but is decreased in distal axons, of Niemann-Pick C1-deficient neurons. J Neurochem 83:1154-1163.

Lang T, Bruns D, Wenzel D, Riedel D, Holroyd P, Thiele C, Jahn R (2001) SNAREs are concentrated in cholesterol-dependent clusters that define docking and fusion sites for exocytosis. EMBO J 20:2202-2213.

Nicholson AM, Ferreira A (2009) Increased membrane cholesterol might render mature hippocampal neurons more susceptible to $\beta$-amyloidinduced calpain activation and tau toxicity. J Neurosci 29:4640-4651.

Ohno-Iwashita Y, Iwamoto M, Ando S, Mitsui K, Iwashita S (1990) A modified theta-toxin produced by limited proteolysis and methylation: a probe for the functional study of membrane cholesterol. Biochim Biophys Acta 1023:441-448.

Parton RG, Richards AA (2003) Lipid rafts and caveolae as portals for endocytosis: new insights and common mechanisms. Traffic 4:724-738.

Prinetti A, Chigorno V, Prioni S, Loberto N, Marano N, Tettamanti G, Sonnino S (2001) Changes in the lipid turnover, composition, and organization, as sphingolipid-enriched membrane domains, in rat cerebellar granule cells developing in vitro. J Biol Chem 276:21136-21145.

Salin PA, Malenka RC, Nicoll RA (1996) Cyclic AMP mediates a presynaptic form of LTP at cerebellar parallel fiber synapses. Neuron 16:797-803.

Sarna JR, Larouche M, Marzban H, Sillitoe RV, Rancourt DE, Hawkes R (2003) Patterned Purkinje cell degeneration in mouse models of Niemann-Pick type C disease. J Comp Neurol 456:279-291.

Sawamura N, Gong JS, Chang TY, Yanagisawa K, Michikawa M (2003) Promotion of tau phosphorylation by MAP kinase Erk1/2 is accompanied by reduced cholesterol level in detergent-insoluble membrane fraction in Niemann-Pick C1-deficient cells. J Neurochem 84:1086-1096.

Shmygol A, Noble K, Wray S (2007) Depletion of membrane cholesterol eliminates the $\mathrm{Ca} 2+$-activated component of outward potassium current and decreases membrane capacitance in rat uterine myocytes. J Physiol 581:445-456.

Taverna E, Saba E, Rowe J, Francolini M, Clementi F, Rosa P (2004) Role of lipid microdomains in P/Q-type calcium channel (Cav2.1) clustering and function in presynaptic membranes. J Biol Chem 279:5127-5134.

Thiele C, Hannah MJ, Fahrenholz F, Huttner WB (2000) Cholesterol binds to synaptophysin and is required for biogenesis of synaptic vesicles. Nat Cell Biol 2:42-49.

Toselli M, Biella G, Taglietti V, Cazzaniga E, Parenti M (2005) Caveolin-1 expression and membrane cholesterol content modulate N-type calcium channel activity in NG108-15 cells. Biophys J 89:2443-2457.

Waheed AA, Shimada Y, Heijnen HF, Nakamura M, Inomata M, Hayashi M, Iwashita S, Slot JW, Ohno-Iwashita Y (2001) Selective binding of perfringolysin $\mathrm{O}$ derivative to cholesterol-rich membrane microdomains (rafts). Proc Natl Acad Sci U S A 98:4926-4931.

Wang PY, Weng J, Anderson RG (2005) OSBP is a cholesterol-regulated scaffolding protein in control of ERK 1/2 activation. Science 307:14721476.

Wasser CR, Ertunc M, Liu X, Kavalali ET (2007) Cholesterol-dependent balance between evoked and spontaneous synaptic vesicle recycling. J Physiol 579:413-429.

Xia F, Gao X, Kwan E, Lam PP, Chan L, Sy K, Sheu L, Wheeler MB, Gaisano HY, Tsushima RG (2004) Disruption of pancreatic beta-cell lipid rafts modifies Kv2.1 channel gating and insulin exocytosis. J Biol Chem 279:24685-24691.

Zamir O, Charlton MP (2006) Cholesterol and synaptic transmitter release at crayfish neuromuscular junctions. J Physiol 571:83-99.

Zhang J, Xue R, Ong WY, Chen P (2009) Roles of cholesterol in vesicle fusion and motion. Biophys J 97:1371-1380. 